\title{
MUSEOLOGIA\&INTERD|SCIPLINARIDADE
}

\section{PROGRAYM DE POS-GRADUACTO}

EM CERCLA DA INFORRACLO

DA URIVERSIDADE DE BRASILLA 



\section{Universidade de Brasília}

Faculdade de Ciência da Informação

\section{Museologia \& Interdisciplinaridade}

Publicação do Programa de Pós-Graduação em

Ciência da Informação - UnB 


\section{Museologia \& Interdisciplinaridade}

Publicação do Programa de Pós-Graduação em

Ciência da Informação - UnB

PPGCINF/FCI/ UnB

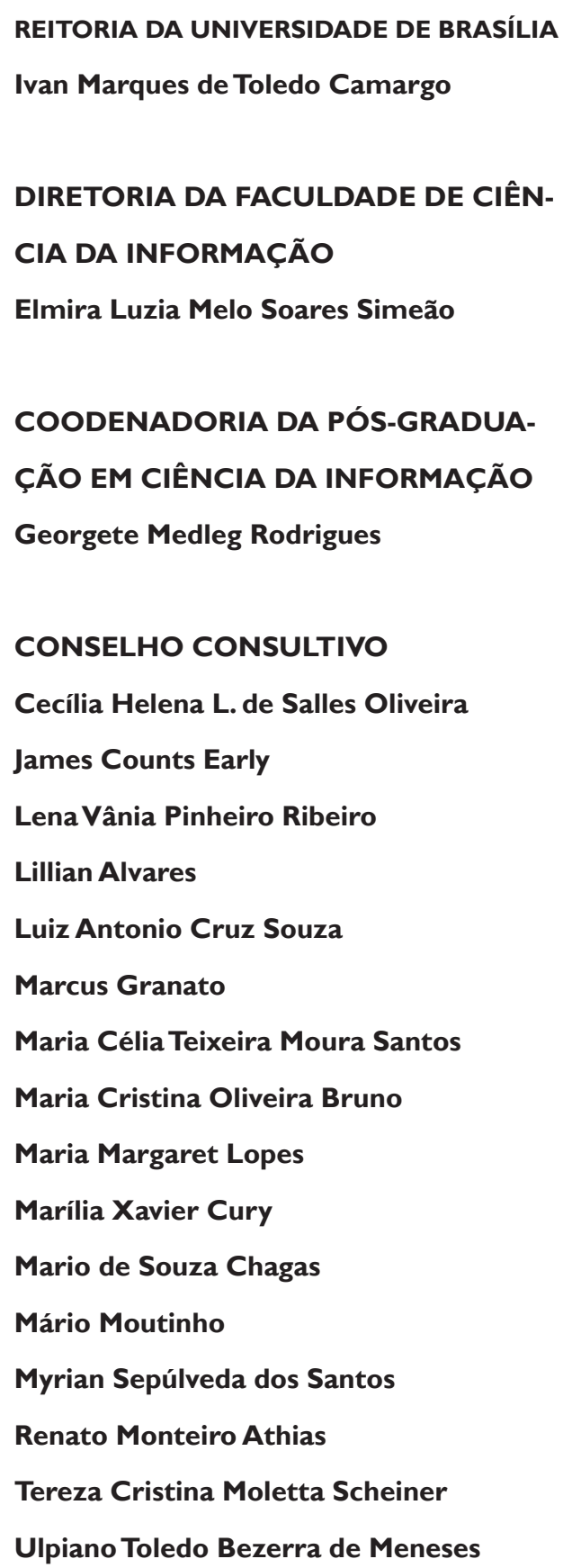

COMISSÃO EDITORIAL

Celina Kuniyoshi

Deborah Silva Santos

Elizângela Carrijo

Luciana Sepúlveda Köptcke

Marijara Souza Queiroz

Monique Batista Magaldi

Silmara Küster de Paula Carvalho

\section{EDITORES}

Ana Lúcia de Abreu Gomes

Andrea Fernandes Considera

Emerson Dionisio Gomes de Oliveira

\section{SECRETARIA}

Vivian Miatello

\section{PROJETO GRÁFICOI}

EDITORAÇÃO ELETRONICA

Núcleo de Editoração e Comunicação/FCI

Cláudia Neves Lopes

Bruna Ribeiro de Freitas

CAPA

André Maya Monteiro 


\section{Universidade de Brasília \\ Faculdade de Ciência da Informação}

\section{Museologia \& Interdisciplinaridade}

Publicação do Programa de Pós-Graduação em

Ciência da Informação - UnB

$n^{\circ}$ 7, Vol. 4, 2015

ISSN 2238-5436 
Museologia \& Interdisciplinaridade

Programa de Pós-Graduação em Ciência da Informação (PPGCInf)

Faculdade de Ciência da Informação ( $\mathrm{FCl}$ ),

Universidade de Brasília

Edifício da Biblioteca Central (BCE),

Entrada Leste, Mezanino, Sala 21 I

Campus Universitário Darcy Ribeiro,Asa Norte, Brasília

CEP: 70910-900

e-mail: revistami@unb.br ;

Contribuições devem ser submetidas pelo site:

http://seer.bce.unb.br/index.php/museologia

Todos os direitos reservados

A reprodução não autorizada desta publicação, no todo ou em parte, constitui violação dos direitos autorais (Lei no 9.610 ).

Dados Internacionais de Catalogação na Publicação (CIP)

Museologia e interdisciplinaridade: publicação eletrônica do Programa de Pós-graduação em Ciência da Informação. Universidade de Brasília. Faculdade de Ciência da Informação. - v.4, n.7 (2015) - Brasília: UnB/FCI, 2015-

Semestral

Resumo em português e inglês.

Disponível no SEER: http://seer.bce.unb.br/index.php/museologia

ISSN 2238-5436

1. Museologia. 2. Patrimônio e memória. Artes Visuais. Antropologia. História Interdisciplinaridade em Museologia. I. Universidade de Brasília. Programa de Pós-graduação em Ciência da Informação. Faculdade de Ciência da Informação.

CDU: 069.01(051) 


\section{SUMÁRIO}

EDITORIAL

DOSSIÊ: COMUNICAÇÃO, PÚBLICO E RECEPÇÃO

ATENÇÕES E VISÕES NA AMPLITUDE E DIVERSIDADE MUSEOLÓGICA

Marilia Xavier Cury

REPRESENTACIÓN, POLISEMIAY MUSEOS

Maria Marta Reca

EL MUSEO DE LOS VISITANTES

Silvia Alderoqui

TENSÕES SOBRE A CONSTRUCÃO NARRATIVA DAS HISTÓRIAS INDÍGENAS NO MÚSEU

Louise Prado Alfonso

Márcia Lika Hattori

COMUNICAÇÃO, MEDIAÇÃO E MARKETING

François Mairesse

MUSEUS DE HISTÓRIA, PRODUÇÃO DE CONHECIMENTOS E COMUNICAÇÃO: QUESTÕES PARA DEBATE

Cecília Helena de Salles Oliveira

QUANDO AS MUSAS VESTEM O HÁBITO DIÁLOGO ENTRE ANTROPOLOGIA, MUSEOLOGIA E HISTÓRIA À SOLEIRA DOS MUSEUS MISSIONÁRIOS

Aramis Luis Silva

A BIENAL DE SÃO PAULO, O DEBATE ARTÍSTICO
DOS ANOS I 950 E ACONSTITUIÇÃO DO
PRIMEIRO MUSEU DE ARTE MODERNA DO BRASIL
Ana Gonçalves Magalhães

THE UBIQUITOUS MUSEUM EXACT IMAGINATION, SYNCRETIC SUBJECT, PERFORMATIVE METROPOLIS, MOVING CONSTELLATION

Masimo Canevacci

CARTOGRAFANDO O RIO DE JANEIRO E SEUS MUSEUS:

NOTAS SOBRE UMA "ETNOGRAFIA

AUDIOVISUAL DE PERCURSOS”

Regina Abreu

POLÍTICA DE ACESSIBILIDADE COMUNICACIONAL EM MUSEUS: PARA QUÊ E PARA QUEM?

Amanda Pinto da Fonseca Tojal

INCLUSÃO SOCIAL E A AUDIÊNCIA ESTIMULADA

EM UM MUSEU DE CIÊNCIA

Sibele Cazelli,

Carlos Alberto Quadros Coimbra,

Isabel Lourenço Gomes,

Maria Esther Valente 
O IMAGINÁRIO SOBRE O INDÍGENA: UMA EXPERIÊNCIA DE APRENDIZAGEM SIGNIFICATIVA NO MUSEU DE ARQUEOLOGIA E ETNOLOGIA DA USP

Camilo de Mello Vasconcellos

A PARTICIPAÇÃO EM MUSEUS: CONTRIBUIÇÃO DA RECEPÇÃO PARA A MUSEALIZAÇÃO DA ARQUEOLOGIA MARÍTIMA

Cristiane Eugênia Amarante

CURADORIA DO ACERVO PALEONTOLÓGICO DA UNIVERSIDADE FEDERAL DO RIO GRANDE DO SUL

Débora Diniz,

Helena Loewenstein,

Paula C. Dentzien-Dias

FUNDAMENTOS DE UM CAMPO DISCIPLINAR:

PERSPECTIVAS SOBRE O DESENVOLVIMENTO

DA TEORIA DA MUSEOLOGIA NO ÂMBITO DOS

CURSOS DE GRADUAÇÃO DA UNIRIO

Bruno Brulon

A MULTI-TEMPORALIDADE DO MUSEU: MEIO EXPOSITIVO E REALIDADE MISTA

Giovanna Graziosi Casimiro

A EXPERIÊNCIA MUSEAL: DISCUTINDO A RELAÇÃO DOS MUSEUS COM SEUS VISITANTES

NA CONTEMPORANEIDADE

Manuelina Ma. Duarte Cândido

Gabriela Aidar,

Luciana Conrado Martins

CAPA

Ana Ruas 


\title{
EDITORIAL
}

\author{
Ana Lúcia de Abreu Gomes \\ Emerson Dionisio Gomes de Oliveira
}

Elegemos duas palavras incontornáveis para esse sétimo número da Museologia \& Interdisciplinaridade: generosidade e confiança. Marília Xavier Cury confiou nesta publicação desde o seu primeiro número em 20I2. Tendo seus artigos como os mais acessados, sua confiança nesta jovem revista se desdobrou em generosidade, ao aceitar organizar o dossiê intitulado Comunicação, recepção e público que ora apresentamos.

São treze artigos que discutem o tema sob a perspectiva dos caminhos percorridos por essa relação recentemente reconhecida no campo e que, por isso, nos falam de experiências que nos desafiam à reflexão a todo momento. $O$ desafio, não de esgotar o tema, mas de explorá-lo em suas diferentes possibilidades de abordagem foi posto a diversos autores convidados pela Prof. ${ }^{a}$ Marília Xavier Cury que os reuniu, no dossiê que ora se apesenta, adensando o debate acerca do tema.

São apresentadas perspectivas de atualização dos museus por meio de diferentes atividades expositivas, por meio da compreensão desses espaços como condição de possibilidade do debate, do conflito, enfim do protagonismo do público. Há ainda os artigos que abordam temas consolidados como acervo e acessibilidade na perspectiva da comunicação e a discussão da comunicação, recepção e público em museus indígenas um de seus atuais campos de pesquisa.

Os quatro artigos avulsos que acompanham o dossiê deste número nos apresentam as dificuldades que envolvem as atividades de curadoria diante de um acervo delicado: o acervo paleontológico do Rio Grande do Sul. Na sequencia, Bruno Brulon e Elizabeth Mendonça nos convidam a conhecer os diferentes projetos pedagógicos adotados ao longo dos quase quarenta anos do curso de graduação da escola de Museologia da UniRio e suas relações com a Teoria Museológica. Retomando o debate presente nos artigos do Dossiê temos o artigo de Giovanna Graziosi Casimiro que se propõe a discutir o uso de ferramentas computacionais na construção do espaço expositivo e o artigo de Manuelina Maria Duarte Cândido, Gabriela Aidar e Luciana Conrado Martins debatendo a experiência museal e o papel dos visitantes.

Sempre no intuito de contribuir para a qualificação do campo, desejamos a todos profícuos momentos de reflexão.

Emerson Dionisio Gomes de Oliveira

Ana Lúcia de Abreu Gomes

Andrea Fernandes Considera 
10

옴

चั

를

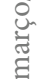




\section{NOSSOS PARECERISTAS}

A publicação de revista Museologia e Interdisciplinariedade não seria possível sem a constituição de um corpo de pareceristas que atuam como avaliadores dos trabalhos submetidos à Revista. Um trabalho coletivo que agrega pesquisadores de diferentes áreas do conhecimento. Pesquisadores que gentilmente colaboraram de maneira voluntária. Agradecemos nominalmente aos colegas que atenderam a nossa solicitação e tornaram-se parte integrante da história dessa jovem publicação:

Adriana Mortara Almeida (Museu Histórico do Instituto Butantan)

Alda Lucia Heizer (Inst. de Pesquisas Jardim Botânico do Rio de Janeiro-JBRJ)

Ana Lúcia de Abreu Gomes (UnB)

Ana Maria Dalla Zen (UFRGS)

Andréa Fernandes Considera (UnB)

Camila Azevedo de Moraes Wichers (UFG)

Camilo de Mello Vasconcellos (USP)

Carlos Alberto Ávila Araujo (UFMG)

Cátia Rodrigues Barbosa (UFMG)

Elizabete de Castro Mendonça (UFS)

Emanuel Sousa Ribeiro (UFPE)

Emerson Dionisio Gomes de Oliveira (UnB)

Fabíola Andreá Silva (MAE-USP)

Francisco Sá Barreto (UFPE)

José Cláudio Alves de Oliveira (UFBA)

Karla Estelita Godoy (UFF)

Ligia Maria Arruda Café (UFSC)

Lillian Maria Araújo de Rezende Alvares (UnB)

Luciana Ferreira da Costa (UFPB)

Luciana Sepúlveda Köptcke (UnB/Fiocruz)

Luiz Antonio Cruz Souza (UFMG)

Maria Esther Alvarez Valente (MAST/UNIRIO)

Maria Júlia Estefânia Chelini (UnB)

Maria Margaret Lopes (UnB/Unicamp)

Martha Marandino (USP)

Milton Terumitsu Sogabe (Unesp)

Monique Bastista Magaldi (UnB)

Renato Athias (UFPE)

Robson Xavier da Costa (UFPB/UFPE)

Sidélia Santos Teixeira (UFBA)

Silmara Küster de Paula Carvalho (UnB)

Suzana Cesar Gouveia Fernandes (Instituto Butantan)

Thérèse Hofmann Gatti Rodrigues da Costa (UnB)

Valdir José Morigi (UFRGS)

Zita Rosane Possamai (UFRGS) 
12 